\title{
Group-Level Emotion Recognition using Deep Models with A Four-stream Hybrid Network
}

\author{
Ahmed Shehab Khan \\ University of South Carolina \\ Columbia, South Carolina \\ akhan@email.sc.edu \\ Zibo Meng \\ InnoPeak Technology Inc. \\ Palo Alto, California \\ mzbo1986@gmail.com
}

\author{
Zhiyuan Li \\ University of South Carolina \\ Columbia, South Carolina \\ zhiyuanl@email.sc.edu \\ James O’Reilly \\ University of South Carolina \\ Columbia, South Carolina \\ oreillyj@email.sc.edu
}

\author{
Jie Cai \\ University of South Carolina \\ Columbia, South Carolina \\ jcai@email.sc.edu \\ Yan Tong \\ University of South Carolina \\ Columbia, South Carolina \\ tongy@cse.sc.edu
}

\begin{abstract}
Group-level Emotion Recognition (GER) in the wild is a challenging task gaining lots of attention. Most recent works utilized two channels of information, a channel involving only faces and a channel containing the whole image, to solve this problem. However, modeling the relationship between faces and scene in a global image remains challenging. In this paper, we proposed a novel face-location aware global network, capturing the face location information in the form of an attention heatmap to better model such relationships. We also proposed a multi-scale face network to infer the group-level emotion from individual faces, which explicitly handles high variance in image and face size, as images in the wild are collected from different sources with different resolutions. In addition, a global blurred stream was developed to explicitly learn and extract the scene-only features. Finally, we proposed a four-stream hybrid network, consisting of the face-location aware global stream, the multi-scale face stream, a global blurred stream, and a global stream, to address the GER task, and showed the effectiveness of our method in GER sub-challenge, a part of the six Emotion Recognition in the Wild (EmotiW 2018) [10] Challenge. The proposed method achieved $65.59 \%$ and $78.39 \%$ accuracy on the testing and validation sets, respectively, and is ranked the third place on the leaderboard.
\end{abstract}

\section{KEYWORDS}

Emotion Recognition; Group-level emotion recognition; Affect Analysis; Attention Heatmap

\section{ACM Reference Format:}

Ahmed Shehab Khan, Zhiyuan Li, Jie Cai, Zibo Meng, James O’Reilly, and Yan Tong. 2018. Group-Level Emotion Recognition using Deep Models with A Four-stream Hybrid Network. In 2018 International Conference on Multimodal Interaction (ICMI '18), October 16-20, 2018, Boulder, CO, USA. ACM, New York, NY, USA, 7 pages. https://doi.org/10.1145/3242969.3264987

Permission to make digital or hard copies of all or part of this work for personal or classroom use is granted without fee provided that copies are not made or distributed for profit or commercial advantage and that copies bear this notice and the full citation on the first page. Copyrights for components of this work owned by others than ACM must be honored. Abstracting with credit is permitted. To copy otherwise, or republish, to post on servers or to redistribute to lists, requires prior specific permission and/or a fee. Request permissions from permissions@acm.org.

ICMI '18, October 16-20, 2018, Boulder, CO, USA

(c) 2018 Association for Computing Machinery.

ACM ISBN 978-1-4503-5692-3/18/10 . \$ \$15.00

https://doi.org/10.1145/3242969.3264987

\section{INTRODUCTION}

Emotion recognition has been widely studied because of its crucial role in a wide range of applications in human-computer interaction, security, and affective computing [31]. Enormous efforts have been devoted to emotion recognition for individuals in decades. Most recently, thanks to easy access to cameras and a large number of active social network users, an exponential growth of available images taken in natural setting has been observed. The availability of large-scale natural data has shifted the focus of emotion recognition for individual subjects to in-the-wild setting as well as opened an opportunity for researchers to work on Group-level Emotion Recognition (GER). GER has immense importance in various applications such as image retrieval, early event prediction, surveillance, and image set visualization [9]. While the challenges of emotion recognition for individuals still persist, like head pose, occlusions, and illumination variations, GER adds additional challenges like scene variations and interactions between multiple persons in the group [28].

The state-of-the-art approaches for the GER task [14, 35, 38] employed a combination of top-down and bottom-up approaches. The bottom-up approach entails detecting faces from the image, extracting features and performing expression recognition from each of them, and finally combining individual predictions for group-level prediction. The top-down approach extracts features and makes a prediction from the whole image directly [9]. In this work, we proposed a hybrid GER framework, which consists of four independent streams. First, a multi-scale face stream was proposed to handle large scale-variations of input face images. Second, a novel face-location aware global stream was proposed to model the relationship between faces and scene by incorporating face location and size information as an attention heatmap to the whole image. Third, a global stream was utilized to make the group-level prediction directly from the whole image. Finally, a global blurred stream was developed to explicitly learn the scene-only features by disentangling the scene features from the facial features.

In summary, our major contributions are:

- A hybrid framework for the GER task;

- A novel face-location aware global stream to incorporate face related information in the global stream;

- A multi-scale face stream to handle large variations in input image size and face size; and 

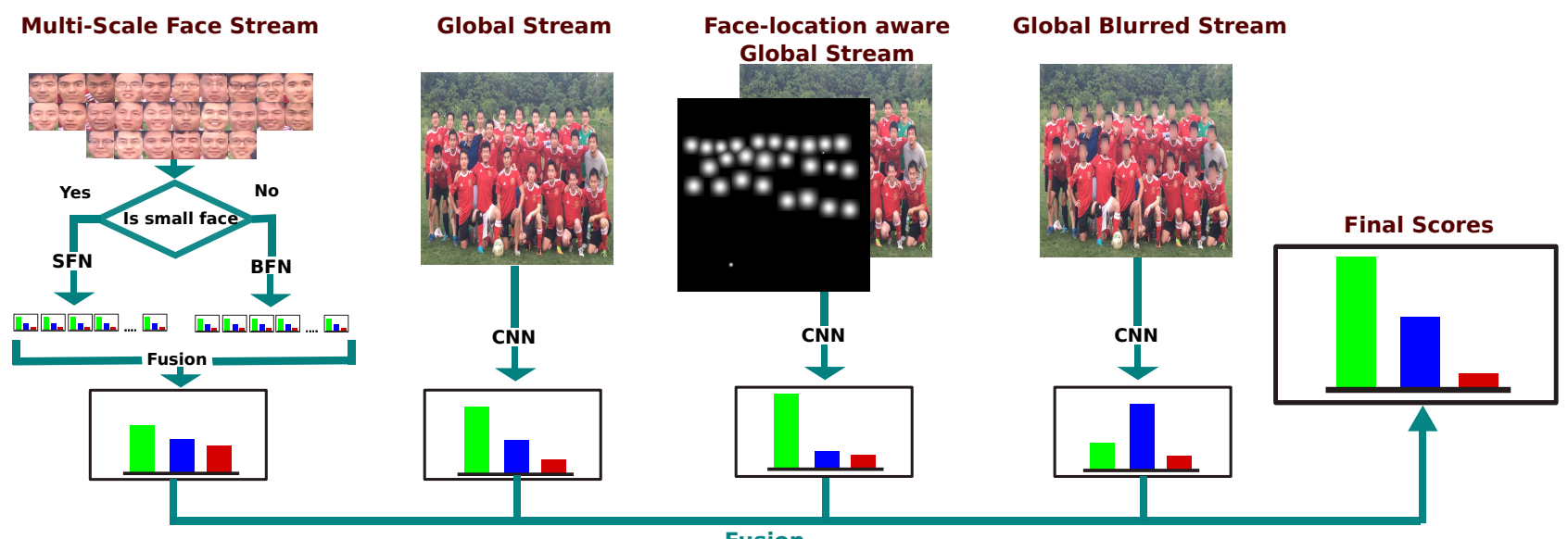

Fusion

Figure 1: Pipeline of our proposed four-stream hybrid network. In the multi-scale face stream, SFN and BFN stand for Small Face Network and Big Face Network, respectively.

- A global blurred stream to extract scene-only features from the whole image.

\section{RELATED WORK}

Emotion recognition for individuals is an essential component for understanding GER. One essential step is to extract discriminative features for different emotion classes. These features can be either hand-crafted or learned from data. Some effective handcrafted features are Gabor wavelets [3], Scale Invariant Feature Transform (SIFT) features [6, 42], histogram of Oriented Gradients (HOG) [2], histograms of Local Binary Patterns (LBP) [20, 36], histograms of Local Phase Quantization (LPQ) [18], histograms of Local Gabor Binary Patterns (LGBP) [27] and the spatiotemporal extensions of the aforementioned 2D features [19, 21, 32, 41, 44, 45]. More recently, features have been learned either unsupervised by sparse coding $[26,30,46]$ or supervised by deep learning $[4,5,11,12,15$, $16,24,25,39,40]$.

GER has gained more and more interest in recent years with the growth of public datasets. Dhall et al. [8] developed an automatic framework considering both global and local emotional attributes for happiness intensity analysis for groups of people in a social event and published the HAPPEI database. Several methods for group happiness intensity estimation have been developed for Group based Emotion Recognition in the Wild (EmotiW) 2016. The winning team [22] extracted face related features using ResNet and used CENTRIST as global features, and then, employed both face and global features as input to an LSTM. The team ranked second [37] used landmark based geometrical facial features to train a regression model predicting happiness. The third team [34] employed an LSTM network trained on CNN features along with handcrafted features like LBP and dense SIFT features.

Most recently, Dhall et al. [9] presented a Multiple Kernel Learning based hybrid GER inference model and published Group Affect Database, which contains images of a group of people at a social event labeled as Positive, Negative or Neutral. Since then, research has been done on group affect analysis and benchmarked in EmotiW 2017. The winning team [35] and the third team [38] utilized two streams of $\mathrm{CNN}$, one for individual emotion recognition and the other for global-level emotion recognition, which are combined to get the final prediction of GER. The second team [14] developed a hybrid network that can utilize global scene features, skeleton features of the group, and also local facial features.

\section{METHODOLOGY}

As depicted in Fig. 1, our proposed hybrid GER framework contains four independent streams. The first stream, i.e., a multi-scale face stream, infers the group-level emotion from individual faces extracted from the whole image. The second stream, i.e., the global stream, extracts information from the whole image directly. The third stream, i.e., the face-location aware global stream, not only extracts information from the whole image but also incorporates the information of face size and location as additional input in form of attention heatmaps. The last stream, i.e., the global blurred stream, only utilizes the scene information by removing detailed face information from the image. In the following, we will describe each stream of the hybrid network in detail.

\subsection{A Multi-scale Face Stream}

The multi-scale face stream (MS-FS) focuses on estimating the group-level emotion from individual faces in the image.

Face Detection: We used MTCNN [43] to detect faces from the input image, which is three-stage cascaded CNN networks for joint face detection and landmark localization. The detected faces were then cropped and aligned by a similarity transform.

Multi-Scale Face Stream: The aligned faces were used to train $\mathrm{CNN}$ networks for facial expression recognition. However, the extracted faces are of different sizes, ranging from $7 * 7$ to $1590 * 1590$ in the original images and are mostly concentrated around $20 * 20$ as shown in Fig. 2. Having this large variation in face size, it is difficult to choose an optimal input face size for a $\mathrm{CNN}$ network since the input face images will be significantly blurred due to up-sampling the low-resolution images. To alleviate this problem, we proposed to build a multi-scale face stream consisting of two networks for two different ranges of input face sizes. The first one is a Small Face 


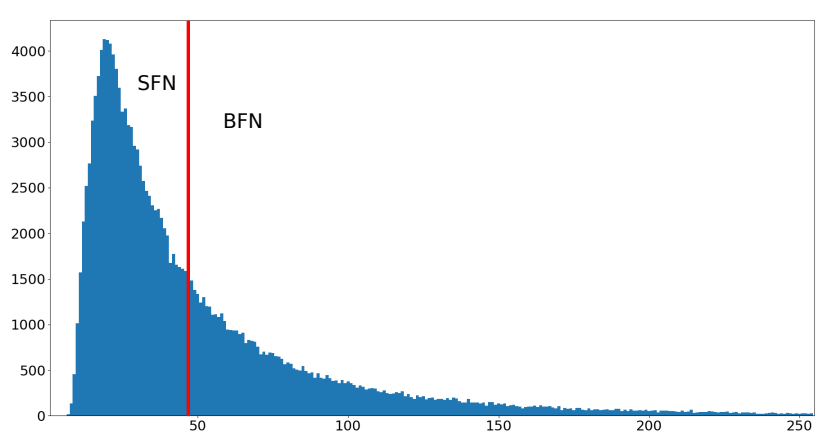

Figure 2: A histogram of extracted face areas. The red line shows the threshold for sending the images to the SFN and the BFN, respectively.

Network (SFN), which takes inputs of size $24 * 24$, and the other is a Big Face Network (BFN), which takes input size of $48 * 48$. Faces with a size smaller than $48 * 48$ are used as input of the SFN, while the larger ones are used by the BFN. We chose $48 * 48$ as the threshold empirically because various previous work [13,25] has shown it to be an effective input size for facial expression recognition that often employs high-resolution face images. In this work, 34 and 18 layer Residual Networks [17] were employed as the architecture of SFN and BFN, respectively. We chose a relatively deeper structure for the SFN, as it has more sample images.

Specifically, we made two major changes in the Residual Network architecture to accommodate the various input sizes. First, we changed the stride of the first max-pooling layer from 2 to 1 to avoid down-sampling in the early stage of the network, since the input sizes are already very small, i.e, 24 and 48 pixels, compared to the standard input, i.e., 224 pixels, in the original Residual Network. Second, the change in input size results in different size filter responses after the final convolution layer. Hence, we changed the final average pooling kernel to be $2 * 2$ for the SFN and $3 * 3$ for the BFN.

Group-level Emotion Prediction from the Face Stream: The group-level prediction was calculated as a weighted average of the predictions from individual faces. Since the faces in focus are usually larger and are the most influential characters in the group, we proposed to estimate the weights based on the face sizes as in Eqn. 1. Furthermore, the proposed weighting strategy can alleviate the effect of unreliable predictions from smaller background faces by assigning lower weights to smaller faces.

$$
\begin{array}{r}
w_{i}=\frac{\sqrt{a_{i}}}{\sum_{x} \sqrt{a_{x}}} \\
p_{m s-f s}=\sum_{i} w_{i} * p_{i}
\end{array}
$$

where $a_{i}$ is the area of the $i$ th face in an image, $w_{i}$ is the fusion weight assigned to the $i$ th face, $p_{i}$ is the individual prediction score of the $i$ th face, and $p_{m s-f s}$ is the group-level prediction score of the whole image from the face stream.

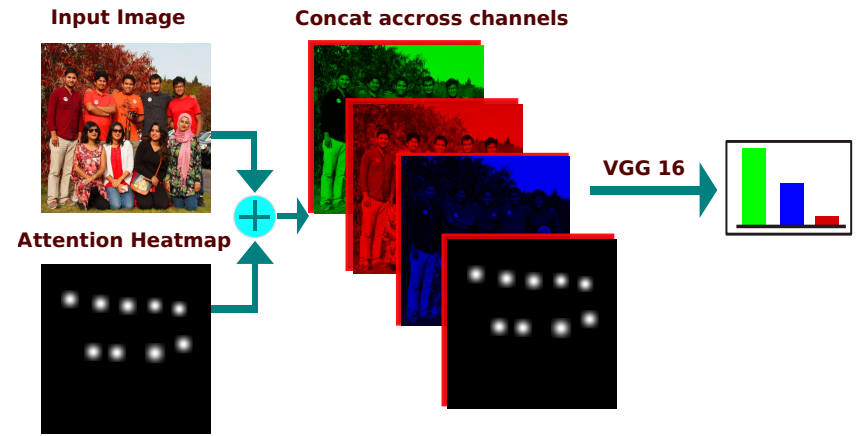

Figure 3: An illustration of the face-location aware global stream. Note that heatmaps are used as an additional input along with the three color channels of the input images.

\subsection{A Global Stream}

A global stream (GS) was employed to recognize the group-level affect from the whole image. This stream is necessary and has been demonstrated to be effective by early studies $[35,38]$, since the face stream only considers the face-related features while ignores the scene. Moreover, the face stream lacks information to model the spatial relationships and interactions between faces. The global stream learns the discriminative features from the whole image and models the global context. In this work, a 16-layer VGG network [33] was used for the global stream. Specifically, we fine-tuned a pre-trained model originally trained on ImageNet [7] 1000 class classification task.

\subsection{A Face-location Aware Global Stream}

In order to get a picture of the group-level affect, the global stream needs to be aware of faces present in the image and their relative locations together with the scene information. However, the aforementioned global stream does not have any constraints enforcing to learn face locations and sizes, which we consider as critical information to understand group-level emotion. To handle this issue, we proposed a novel face-location aware global stream (FLA-GS). As shown in Fig. 3, a face-attention heatmap is used as an input channel to this stream in addition to the three color channels of the whole image. This additional heatmap enables the network to give special attention to face regions as well as to learn relationships between faces.

Specifically, the heatmap is generated by creating a 2D Gaussian mask for each face in the image. The 2D Gaussian mask is centered at the center of the face bounding box, and the standard deviation of the distribution is set as one-sixth of the face height. Let $\left(c_{x, k}, c_{y, k}\right)$ be the center of the $k$ th detected face region, the corresponding $2 \mathrm{D}$ Gaussian mask is calculated as:

$$
H_{k}\left(i+c_{x}, j+c_{y}\right)= \begin{cases}e^{-\frac{\left(i^{2}+j^{2}\right)}{2 \sigma^{2}}} & \text { if }\|i\|,\|j\| \leq 3 \sigma \\ 0 & \text { otherwise. }\end{cases}
$$

For this stream, we also used a 16-layer VGG network, but modified the first convolution layer to take an input of 4 channels, where 
the first 3 channels are the three color channels of the input image and the fourth channel is the generated attention heatmap containing information about face location and size.

\subsection{A Global Blurred Stream}

A quick glimpse into the training dataset gave us an impression that although the faces in the image convey the major information, the scene alone plays an important role as well. For example, the same faces in a convocation and at a funeral would convey totally different emotions to the viewers of the image. However, we did not explicitly constrain the global stream to learn scene related features only. Hence, we proposed a global blurred stream (GBS), in which the faces are blurred with a zero-mean Gaussian blurring kernel. The standard deviation of the Gaussian kernel is set as one-sixth of the face height. This channel was employed in order to disentangle the scene related features from the facial features.

\subsection{Fusion of the Four Streams}

Having predictions from individual streams, we combined them by a weighted average of the individual streams' prediction scores ${ }^{1}$ as below:

$$
\begin{array}{r}
p=w_{m s-f s} * p_{m s-f s}+w_{g s} * p_{g s} \\
+w_{f l a-g s} * p_{f l a-g s}+w_{g b s} * p_{g b s} \\
w_{m s-f s}+w_{g s}+w_{f l a-g s}+w_{g b s}=1
\end{array}
$$

where $p_{m s-f s}, p_{g s}, p_{f l a-g s}$, and $p_{g b s}$ are the prediction scores of the multi-scale face stream, global stream, face-location aware global stream, and global blurred stream, respectively, and $w_{m s-f s}$, $w_{g s}, w_{f l a-g s}$, and $w_{g b s}$ are corresponding fusion weights.

\section{EXPERIMENTS}

\subsection{Experimental Dataset}

The dataset used for EmotiW 2018 GER sub-challenge [10] was collected from Google Images and Flickr based on keyword search The keywords used for search correspond to different events, both happy and sad. Some examples of keywords used are festival, party, silent protest, violence, etc. The dataset was divided into three partitions, i,e. Train (9815 samples), Val (4346 samples), and Test (3011 samples). Each sample image in the dataset belongs to one of the three classes: Positive, Negative and Neutral.

\subsection{Implementation Details}

We used PyTorch [29] as a deep learning framework for all of our experiments. By default model/parameters, we refer to PyTorch defaults.

For the multi-scale face stream, we used a public implementation of MTCNN framework [1] to detect faces. Then, we adapted ResNet 18 for the BFN, and ResNet34 for the SFN, while changing the default architectures to accommodate the input sizes as described in Sec. 3.1. The SFN and BFN were first trained on the RAF-DB [23] and FER-2013 [13] databases and then fine tuned on EmotiW 2018 dataset [10]. For pretraining using the datasets containing 7 expression categories, we changed labels of Happiness, Surprise to

\footnotetext{
${ }^{1}$ weights are determined by a grid search on the validation set.
}

Positive, and Angry, Disgust, Fear, and Sadness to Negative to match the target tasks.

In our training process, every image went through a series of the following transformations in the order: resizing, random rotation, random horizontal flipping, random cropping, and normalization, for data augmentation. Specifically, for BFN, images were resized to $60 * 60$ and cropped to $48 * 48$; and for SFN, images were resized to $28 * 28$ and cropped to $24 * 24$. For both networks, random rotation from $-3^{\circ}$ to $+3^{\circ}$ was used.

In each epoch, we sampled 15, 000 images with each class having equal probability for being chosen. The networks were trained for 60 epochs, with a starting learning rate of 0.01 while the learning rate was multiplied by 0.1 every 10 epochs. We used stochastic gradient descent (SGD) as the optimizer with 0.9 momentum and $1 e^{-4}$ weight decay. For prediction, we chose a maximum of 10 faces for each of SFN and BFN if available. If more than 10 faces were detected, we chose the ones with maximum detection confidence and completely ignored those less than $12 * 12$ or detected with confidence less than 0.90 . Furthermore, we constructed an ensemble with 10 networks, which were trained independently from 10 independently pre-trained networks, and calculated the average as the final prediction score.

For all the global streams, i.e, GS, FLA-GS, and GBS, we started with a VGG 16 network with batch normalization pretrained on ImageNet [7], provided by the PyTorch library. The following transformations were applied to the input images in order: resizing, random rotation, random horizontal flipping, random cropping, and normalization. Images were resized to $256 * 256$ and cropped to $224 * 224$. Rotation range used was $-3^{\circ}$ to $+3^{\circ}$. We trained the networks for 32 epochs over the entire training set, with a starting learning rate of 0.01 while the learning rate was multiplied by 0.1 every 8 epochs. The SGD was used as the optimizer with 0.9 momentum and $1 e^{-4}$ weight decay.

For final prediction of the hybrid network, the images with no detected faces are given prediction scores of 0 for all three classes for MS-FS, FLA-GS, and GBS, which require the detected face bounding boxes, and the final prediction result of group-level emotion completely depends on the GS.

\subsection{Experimental Results and Discussions}

In this section, we discuss the experimental results of our proposed approach. First, the results from the individual streams are presented; and then we present and discuss the result from the hybrid network and analyze the contributions of individual streams towards the final classification result.

Individual stream based experimental results are compared in Table 1 in terms of group-level emotion recognition accuracy on the validation set.

We first evaluated the BFN-ResNet18 with $48 * 48$ input and the SFN-ResNet34 with $24 * 24$, separately. As shown in Table 1 , the two single-scale networks achieved similar group-level recognition performance: $69.72 \%$ (BFN) and $69.77 \%$ (SFN). By considering the variance in scale explicitly, the proposed MS-FS improves the accuracy to $71.41 \%$. Moreover, an ensemble of $10 \mathrm{MS}-\mathrm{FS}$ further improves accuracy to $72.69 \%$. Tables 2,3 , and 4 also report the confusion matrices of the SFN-ResNet34, MS-FS, and ensemble MS-FS, 
respectively. Results of the face streams, both accuracies and confusion matrices, are reported on a subset of validation set with the images containing faces with size larger than $12 * 12$ and detected with more than .90 confidence score.

From Tables 2, 3, and 4, it can be observed that all face streams perform relatively better for the positive and negative classes, but worse in recognizing the neutral class. The reason behind this is the face-stream based group-level emotion recognition is dominated by the facial expressions, while a strong muscular indication on face can be observed in happy, surprised, or angry faces. Hence, a crying face in a festive environment still conveys a negative emotion, and a happy face mostly conveys a positive emotion irrespective of the environment. However, group-level emotion conveyed by neutral faces largely depends on the scene. For example, a neutral face in a meeting conveys neutral group-level emotion, but the same face in a funeral or in a protest would convey negative group-level emotion. Moreover, faces usually occupy less area in the whole image and the face streams utilize low-resolution faces, which impedes the recognition performance of emotion recognition from individual faces. Therefore, it is necessary to combine face-based predictions with scene information from the global streams.

Table 1: Performance comparison of individual streams in terms of group-level emotion recognition accuracy on the validation set

\begin{tabular}{c|c}
\hline Stream & Accuracy \\
\hline Small Face Network (ResNet34) & $69.77 \%$ \\
Big Face Network (ResNet18) & $69.72 \%$ \\
Multi-Scale Face Stream & $71.47 \%$ \\
Ensemble of Multi-Scale Face Stream & $72.69 \%$ \\
Global Stream & $74.87 \%$ \\
Face-location Aware Global Stream & $73.87 \%$ \\
Global Blurred Stream & $61.57 \%$ \\
4-stream hybrid framework & $78.39 \%$
\end{tabular}

Table 2: Confusion matrix of the SFN-ResNet34 on the validation set

\begin{tabular}{cccc}
\hline & Pos & Neu & Neg \\
\hline Pos & 83.98 & 9.89 & 6.13 \\
Neu & 16.65 & 56.61 & 31.67 \\
Neg & 13.10 & 22.89 & 64.01
\end{tabular}

Table 3: Confusion matrix of the MS-FS on the validation set

\begin{tabular}{cccc}
\hline & Pos & Neu & Neg \\
\hline Pos & 83.53 & 9.82 & 6.64 \\
Neu & 11.96 & 58.56 & 29.47 \\
Neg & 10.05 & 22.84 & 67.19
\end{tabular}

The global streams, i.e., GS, FLA-GS, and GBS, achieved an average recognition accuracy of $74.87 \%, 73.87 \%$, and $61.57 \%$, respectively. Tables 5, 6, and 7 also report the confusion matrices of the GS, FLAGS, and GBS, respectively. It can be observed that the GS, taking the
Table 4: Confusion matrix of the ensemble of MS-FSs on the validation set

\begin{tabular}{cccc}
\hline & Pos & Neu & Neg \\
\hline Pos & 84.10 & 8.20 & 7.70 \\
Neu & 10.60 & 59.73 & 29.63 \\
Neg & 10.05 & 20.33 & 69.62
\end{tabular}

input of the whole image, performs the best in terms of the average recognition accuracy. However, the FLA-GS obtained the highest performance on recognizing positive samples by incorporating face location and size information.

While face streams focus entirely on the faces, global streams extract information from the whole image with both faces and scene. Note that both GS and FLA-GS significantly outperform the performance of face streams in terms of average recognition accuracy. Furthermore, the GBS alone can get $61.55 \%$ accuracy on the validation set, which is well above random guess. Therefore, our experiments assert the importance of scene features for group-level emotion recognition.

Table 5: Confusion matrix of the GS on the validation set

\begin{tabular}{cccc}
\hline & Pos & Neu & Neg \\
\hline Pos & 81.34 & 12.88 & 05.78 \\
Neu & 12.79 & 68.57 & 18.64 \\
Neg & 10.80 & 16.49 & 72.71
\end{tabular}

Table 6: Confusion matrix of the FLA-GS on the validation set

\begin{tabular}{cccc}
\hline & Pos & Neu & Neg \\
\hline Pos & 84.96 & 10.18 & 04.86 \\
Neu & 13.92 & 64.52 & 21.56 \\
Neg & 11.18 & 20.52 & 68.31
\end{tabular}

Table 7: Confusion matrix of the GBS on the validation set

\begin{tabular}{cccc}
\hline & Pos & Neu & Neg \\
\hline Pos & 78.52 & 15.41 & 06.07 \\
Neu & 35.97 & 52.85 & 11.18 \\
Neg & 27.94 & 25.54 & 46.43
\end{tabular}

\section{Experimental results of the hybrid framework:}

Each stream has its own advantages. For example, face streams are effective for recognizing emotions involving strong muscular movements associated with emotions such as happiness and anger, while global streams are more capable of recognizing neutral emotion. It is, therefore, necessary to utilize a fusion strategy to take advantage of all streams.

In Table 8 , we demonstrate the improvements gained by adding streams. Starting with the GS as it has the best performance among all streams, we gradually added the MS-FS, FLA-GS, and GBS to show the relative improvement in accuracy. It shows that every additional stream contributes to the overall performance positively. 
In this work, the weights are chosen empirically for MS-FS, GS, FLA-GS, and GBS streams are $0.65,0.12,0.18$ and 0.05 , respectively. Hence, the MS-FS contributed the most, and the GBS with the worst individual performance contributed the least to the final prediction. Tables 9 and 10 also report the confusion matrices of the GS + MS-FS and the GS + MS-FS + FLA-GS networks.

Comparing Tables 5 and 9, we can find that the addition of only face streams (GS + MS-FS) improved the accuracy of positive and negative classes, but the accuracy of neutral class slightly decreases. It is consistent with our observation that faces provides strong emotional cues for positive and negative classes, while struggle with neutral. Table 10 shows that adding the FLA-GS helps to improve the accuracy in all three classes. In addition, incorporating the GBS slightly improves the overall recognition performance, as shown in Table 11.

Table 12 reports the performance of the proposed 4-stream hybrid framework on the test set, which was trained using both training and validation sets. We achieved $65.59 \%$ overall accuracy, $73.93 \%$ for the positive class, $55.34 \%$ for the neutral class, and $64.17 \%$ for the negative class.

Table 8: Effect of individual streams on the overall recognition performance

\begin{tabular}{lc}
\hline Models & Validation Set Accuracy \\
\hline GS & 74.87 \\
GS + MS-FS & 76.83 \\
GS + MS-FS + FLA-GS & 78.20 \\
GS + MS-FS + FLA-GS + GBS & 78.39
\end{tabular}

Table 9: Confusion matrix of GS + MS-FS on the validation set

\begin{tabular}{cccc}
\hline & Pos & Neu & Neg \\
\hline Pos & 86.38 & 09.16 & 04.46 \\
Neu & 13.01 & 67.54 & 19.44 \\
Neg & 10.40 & 16.00 & 73.60
\end{tabular}

Table 10: Confusion matrix of GS + MS-FS + FLA-GS on the validation set

\begin{tabular}{lccc}
\hline & Pos & Neu & Neg \\
\hline Pos & 86.89 & 09.16 & 04.46 \\
Neu & 13.01 & 70.03 & 17.91 \\
Neg & 09.34 & 15.68 & 74.98
\end{tabular}

Table 11: Confusion matrix of the 4-stream hybrid framework on the validation set

\begin{tabular}{lccc}
\hline & Pos & Neu & Neg \\
\hline Pos & 87.64 & 08.64 & 03.72 \\
Neu & 11.99 & 70.76 & 17.25 \\
Neg & 09.67 & 16.57 & 73.76
\end{tabular}

Table 12: Confusion matrix of the 4-stream hybrid framework on the test Set

\begin{tabular}{cccc}
\hline & Pos & Neu & Neg \\
\hline Pos & 73.93 & 10.58 & 15.48 \\
Neu & 24.67 & 55.34 & 19.97 \\
Neg & 15.80 & 22.02 & 64.17
\end{tabular}

\section{CONCLUSION}

This paper presents our approach for the Group Level Emotion Recognition sub-challenge of EmotiW 2018. We proposed a fourstream hybrid network consisting of a novel face-location aware global stream using attention heatmap, a multi-scale face stream that explicitly handles image size variation, a global stream, and a global blurred stream capturing scene-only information. We observed that face information plays the major role for recognizing strong emotion expressions like positive and negative; however, scene information is extremely important for the neutral class. In our future work, we will focus on developing methodologies dealing with hard classes and hard samples.

\section{ACKNOWLEDGEMENT}

This work is supported by National Science Foundation under CAREER Award IIS-1149787.

\section{REFERENCES}

[1] Dan Antoshchenko. 2017. mtcnn-pytorch. https://github.com/TropComplique/ mtcnn-pytorch.

[2] T. Baltrusaitis, M. Mahmoud, and P. Robinson. 2015. Cross-dataset learning and person-specific normalisation for automatic Action Unit detection. In FG, Vol. 6. $1-6$.

[3] M. S. Bartlett, G. Littlewort, M. G. Frank, C. Lainscsek, I. Fasel, and J. R. Movellan. 2005. Recognizing Facial Expression: Machine Learning and Application to Spontaneous Behavior. In CVPR. 568-573.

[4] C. Benitez-Quiroz, Y. Wang, and A. Martinez. 2017. Recognition of Action Units in the Wild with Deep Nets and a New Global-Local Loss. In ICCV. IEEE, 3990-3999.

[5] J. Cai, Z. Meng, A. Khan, Z. Li, J. O'Reilly, and Y. Tong. 2018. Island Loss for Learning Discriminative Features in Facial Expression Recognition. In FG. IEEE, 302-309.

[6] W. Chu, F. De la Torre, and J. Cohn. 2016. Selective transfer machine for personalized facial expression analysis. IEEE T-PAMI (2016).

[7] J. Deng, W. Dong, R. Socher, L.-J. Li, K. Li, and L. Fei-Fei. 2009. ImageNet: A Large-Scale Hierarchical Image Database. In CVPR09.

[8] Abhinav Dhall, Roland Goecke, and Tom Gedeon. 2015. Automatic group happiness intensity analysis. IEEE Transactions on Affective Computing 6, 1 (2015), $13-26$.

[9] Abhinav Dhall, Jyoti Joshi, Karan Sikka, Roland Goecke, and Nicu Sebe. 2015. The more the merrier: Analysing the affect of a group of people in images. In Automatic Face and Gesture Recognition (FG), 2015 11th IEEE International Conference and Workshops on, Vol. 1. IEEE, 1-8.

[10] Abhinav Dhall, Amanjot Kaur, Ronald Goecke, and Tom Gedeon. 2018. EmotiW 2018: Audio-Video, Student Engagement and Group-Level Affect Prediction. In ICMI. ACM.

[11] H. Ding, S. Zhou, and R. Chellappa. 2017. Facenet2expnet: Regularizing a deep face recognition net for expression recognition. In FG. IEEE, 118-126.

[12] Y. Fan, X. Lu, D. Li, and Y. Liu. 2016. Video-based emotion recognition using CNN-RNN and C3D hybrid networks. In ICMI. 445-450.

[13] I. Goodfellow, D. Erhan, P. L. Carrier, A. Courville, M. Mirza, B. Hamner, W. Cukierski, Y. Tang, D. Thaler, D. Lee, et al. 2013. Challenges in representation learning: A report on three machine learning contests. In ICML. Springer, 117124.

[14] X. Guo, L. Polanía, and K. Barner. 2017. Group-level emotion recognition using deep models on image scene, faces, and skeletons. In ICMI. ACM, 603-608.

[15] S. Han, Z. Meng, A. S. Khan, and Y. Tong. 2016. Incremental Boosting Convolutional Neural Network for Facial Action Unit Recognition. In NIPS. 109-117.

[16] S. Han, Z. Meng, J. O'Reilly, J. Cai, X. Wang, and Y. Tong. 2018. Optimizing Filter Size in Convolutional Neural Networks for Facial Action Unit Recognition. CVPR 
(2018).

[17] K. He, X. Zhang, S. Ren, and J. Sun. 2016. Deep residual learning for image recognition. In CVPR. 770-778.

[18] B. Jiang, B. Martinez, M. F. Valstar, and M. Pantic. 2014. Decision level fusion of domain specific regions for facial action recognition. In ICPR. 1776-1781.

[19] B. Jiang, M.F. Valstar, and M. Pantic. 2011. Action Unit detection using sparse appearance descriptors in space-time video volumes. In $F G$.

[20] H. Kaya, F. Gürpinar, S. Afshar, and A. A. Salah. 2015. Contrasting and Combining Least Squares Based Learners for Emotion Recognition in the Wild. In ICMI. 459466.

[21] A. Klaser, M. Marszałek, and C. Schmid. 2008. A spatio-temporal descriptor based on 3d-gradients. In BMVC. $275-1$.

[22] J. Li, S. Roy, J. Feng, and T. Sim. 2016. Happiness level prediction with sequential inputs via multiple regressions. In ICMI. ACM, 487-493.

[23] S. Li, W. Deng, and J. Du. 2017. Reliable Crowdsourcing and Deep LocalityPreserving Learning for Expression Recognition in the Wild. In CVPR.

[24] P. Liu, S. Han, Z. Meng, and Y. Tong. 2014. Facial expression recognition via a boosted deep belief network. In CVPR. 1805-1812

[25] Z. Meng, P. Liu, J. Cai, S. Han, and Y. Tong. 2017. Identity-Aware Convolutional Neural Network for Facial Expression Recognition. In FG. IEEE, 558-565.

[26] M. R. Mohammadi, E. Fatemizadeh, and M. H. Mahoor. 2014. Simultaneous recognition of facial expression and identity via sparse representation. In WACV. 1066-1073.

[27] S. Moore and R. Bowden. 2011. Local binary patterns for multi-view facial expression recognition. CVIU 115, 4 (2011), 541-558.

[28] Wenxuan Mou, Oya Celiktutan, and Hatice Gunes. 2015. Group-level arousal and valence recognition in static images: Face, body and context. In Automatic Face and Gesture Recognition $(F G), 2015$ 11th IEEE International Conference and Workshops on, Vol. 5. IEEE, 1-6.

[29] Adam Paszke, Sam Gross, Soumith Chintala, Gregory Chanan, Edward Yang, Zachary DeVito, Zeming Lin, Alban Desmaison, Luca Antiga, and Adam Lerer. 2017. Automatic differentiation in PyTorch. In NIPS-W.

[30] R. Ptucha, G. Tsagkatakis, and A. Savakis. 2011. Manifold based sparse representation for robust expression recognition without neutral subtraction. In ICCV Workshops. 2136-2143

[31] Evangelos Sariyanidi, Hatice Gunes, and Andrea Cavallaro. 2015. Automatic analysis of facial affect: A survey of registration, representation, and recognition.
IEEE transactions on pattern analysis and machine intelligence 37, 6 (2015), 11131133.

[32] P. Scovanner, S. Ali, and M. Shah. 2007. A 3-dimensional sift descriptor and its application to action recognition. In ACM MM. 357-360.

[33] K. Simonyan and A. Zisserman. 2015. Very Deep Convolutional Networks for Large-Scale Image Recognition. In NIPS.

[34] B. Sun, Q. Wei, L. Li, Q. Xu, J. He, and L. Yu. 2016. LSTM for dynamic emotion and group emotion recognition in the wild. In ICMI. ACM, 451-457.

[35] L. Tan, K. Zhang, K. Wang, X. Zeng, X. Peng, and Y. Qiao. 2017. Group emotion recognition with individual facial emotion CNNs and global image based CNNs. In ICMI. ACM, 549-552.

[36] M. F. Valstar, M. Mehu, B. Jiang, M. Pantic, and K. Scherer. 2012. Meta-analysis of the first facial expression recognition challenge. IEEE T-SMC-B 42, 4 (2012), 966-979.

[37] V. Vonikakis, Y. Yazici, V. Nguyen, and S. Winkler. 2016. Group happiness assessment using geometric features and dataset balancing. In ICMI. ACM, 479-486.

[38] Q. Wei, Y. Zhao, Q. Xu, L. Li, J. He, L. Yu, and B. Sun. 2017. A new deep-learning framework for group emotion recognition. In ICMI. ACM, 587-592.

[39] J. Xue, H. Zhang, and K. Dana. 2018. Deep Texture Manifold for Ground Terrain Recognition. In CVPR.

[40] J. Xue, H. Zhang, K. Dana, and K. Nishino. 2017. Differential angular imaging for material recognition. In CVPR, Vol. 5.

[41] P. Yang, Q. Liu, and Metaxas D. N. 2009. Boosting Encoded Dynamic Features for Facial Expression Recognition. Pattern Recognition Letters 30, 2 (Jan. 2009), $132-139$.

[42] A. Yuce, H. Gao, and J. Thiran. 2015. Discriminant Multi-Label Manifold Embedding for Facial Action Unit Detection. In $F G$.

[43] K Zhang, Z Zhang, Z Li, and Y Qiao. 2016. Joint face detection and alignment using multitask cascaded convolutional networks. IEEE Signal Processing Letters 23, 10 (2016), 1499-1503.

[44] G. Zhao, X. Huang, M. Taini, S. Li, and M. Pietikäinen. 2011. Facial expression recognition from near-infrared videos. F. IVC 29, 9 (2011), 607-619.

[45] G. Zhao and M. Pietiäinen. 2007. Dynamic Texture Recognition Using Local Binary Patterns with an Application to Facial Expressions. IEEE T-PAMI 29, 6 (June 2007), 915-928.

[46] L. Zhong, Q. Liu, P. Yang, J. Huang, and D.N. Metaxas. 2015. Learning Multiscale Active Facial Patches for Expression Analysis. IEEE Trans. on Cybernetics 45, 8 (August 2015), 1499-1510 